\title{
On the detection of a brief signal in the temporal center of a noise masker of varying duration
}

\author{
ELI OSMAN, PI-FONG LIN TZUO, and HUAN-YUAN TZUO \\ Brooklyn College, The City University of New York, Brooklyn, New York 11210
}

\begin{abstract}
The threshold of a brief signal temporally centered in a noise masker first increases and then decreases as the masker is increased in duration. We discuss two explanations for this "overshoot" phenomenon which consider the role of peaks of an exponentially weighted sliding integrator. One such explanation takes into account the finding that the steepness of the psychometric function continues to increase monotonically as its location moves first right and then left.
\end{abstract}

If a click is presented repeatedly in the temporal center of a burst of noise, the click threshold varies nonmonotonically with noise duration. Penner (1975) reported such results and proposed a model to explain the small, but problematic, "overshoot" of the function at about $20 \mathrm{msec}$ (see Penner's Figure 4). We have found similar overshoots (consistently for individual listeners), as shown in Figure 1, for a brief tone-burst signal temporally centered in a broadband noise masker. Here we compare Penner's explanation of the overshoot with our own, which considers the covariation of slopes and thresholds of the psychometric function. Our results for the slopes associated with Figure 1 are shown in Figure 2. Tonal signal duration was $11.5 \mathrm{msec}$, with $2.5-\mathrm{msec}$ rise/ fall times. The noise masker was at a spectrum level of $46 \mathrm{~dB}$ SPL. We used a single-interval (yes-no) psychophysical procedure to generate psychometric functions. These were then fit with the logarithmic form of Egan's expression for d' as a power function of the signal-to-noise ratio (Egan, Lindner, \& McFadden, 1969), to get thresholds (corresponding to $76 \%$ correct in a two-interval forced-choice paradigm) and slopes (corresponding to the exponent of the $d^{\prime}$ equation).

Penner $(1975,1976)$ assumed that detection is based on the output of a short-term averaging mechanism or sliding integrator in which the weighting function is exponential and masker (m) and click (c) amplitudes are considered constant during their time of occurrence. Masker variability was ignored in order to carry through the derivation. Assuming a two-interval forced-choice paradigm, Penner's decision rule involves the comparison of the maximum values of $y(t)$, the output of the integrator, one for masker alone, $y_{m}(t)$, and one for masker plus click, $\mathbf{y}_{\mathbf{m}+\mathrm{c}}(\mathrm{t})$.

This research was supported by grants from the PSC-BHE Research Award Program of the City University of New York and The United States Department of Health, Education and Welfare, P.H.S. (Grant R01 NS10843). $y_{m}(t)$ follows a negatively accelerated exponential course up to maximum at masker termination, and then begins an exponential decay. The maximum of

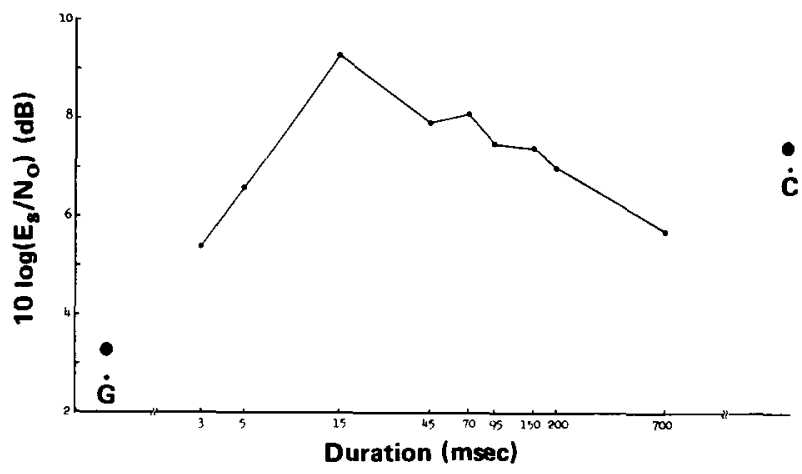

Figure 1. Threshold values for the tonal signal averaged for two listeners (including author H.T.) as a function of the one-sided duration of the noise-fringe. Total noise duration equals the sum of twice the abscissa value and the signal duration (11.5 msec). The point labeled $\mathbf{G}$ is for gated masking where the noise is synchronous with the signal. The point labeled $\mathbf{C}$ is for a continuous masker. A psychometric function was determined for each listener at each value of noise-fringe duration, based on at least 640 trials. The results for the two listeners were then averaged.

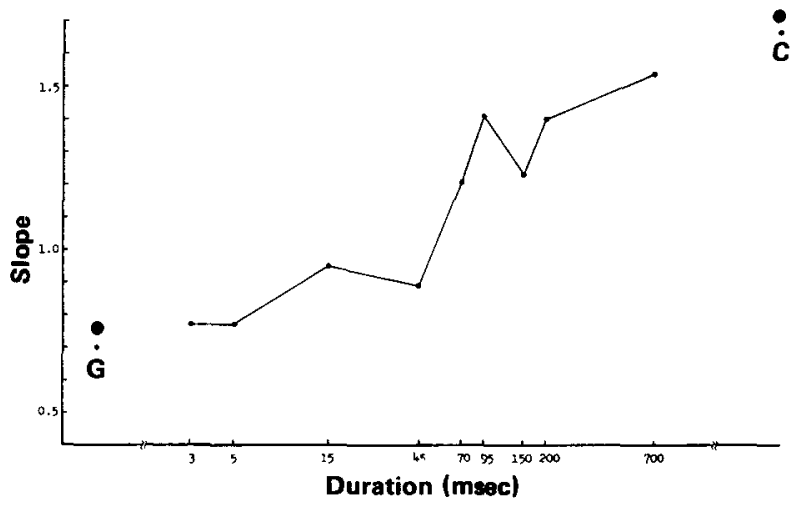

Figure 2. Averaged slopes of the psychometric functions that provided the thresholds of Figure 1. Again, the slope was first determined for each psychometric function for each listener, and then these were averaged for the two listeners. 
$y_{c}(t)$ occurs at termination of the rectangular pulse representing the click, so that the maximum of $y_{m+c}(t)$ $=y_{m}(t)+y_{c}(t)$ must occur somewhere between click termination and masker termination, where $y_{c}(t)$ is decaying as $y_{m}(t)$ is still increasing. Penner shows that the maximum of $y_{m+c}(t)$ occurs either at the termination of the masker, for sufficiently short maskers, or at the termination of the click, for sufficiently long maskers. The sudden transition depends on the time constant of the integrator as well as on the signal-to-masker ratio.

Penner's analysis implies that the listener's decision is based on those two peaks (maxima) of $y(t)$. For long maskers, they would occur at different relative time points within the two observation intervals. The transition presumably occurs at masker durations where the threshold function begins to decline.

The model we first used to evaluate our data is a sliding weighted power integrator which is an extension of the energy detector derived in Green and Swets (1966). The basic equation is similar to Penner's, where the input is a power waveform so that

$$
y(t)=\int_{-\infty}^{t_{m}} w(\tau) x^{2}(t-\tau) d \tau,
$$

where $t_{m}$ is the termination of the masker. Because $\mathrm{w}(\tau)$ is deterministic and we also assumed the exponential form

$$
w(\tau)=e^{-\tau / \xi}, \tau \geqslant 0,
$$

it was possible to derive expressions for conditional means and variances of the underlying conditional probability densities (for noise alone or signal plus noise), using a Fourier series sampling analysis. Then expressions for both the shape and location of the psychometric function were determined using Egan's d' equation. This led to the following conclusions.

For convenience, let $t_{p}$ denote the noise-fringe duration where signal threshold is maximum. A reasonable explanation of the joint variation of psychometric function location and shape when the noise-fringe duration is sufficiently less than $t_{p}$ derives from the weighted power integration model (Tzuo, 1980), and we will not be concerned with that here.

For increasing noise-fringe duration near and beyond $t_{p}$, the fact that the slopes increase significantly beyond unity suggests increasing uncertainty in the detection process. The "M alternatives" model of uncertainty (Green \& Swets, 1966) implies that any increase in the index of uncertainty " $M$ '" forces joint increase in threshold and slope, while for noisefringe durations above $t_{p}$ the empirical threshold slowly decreases as the slope increases.

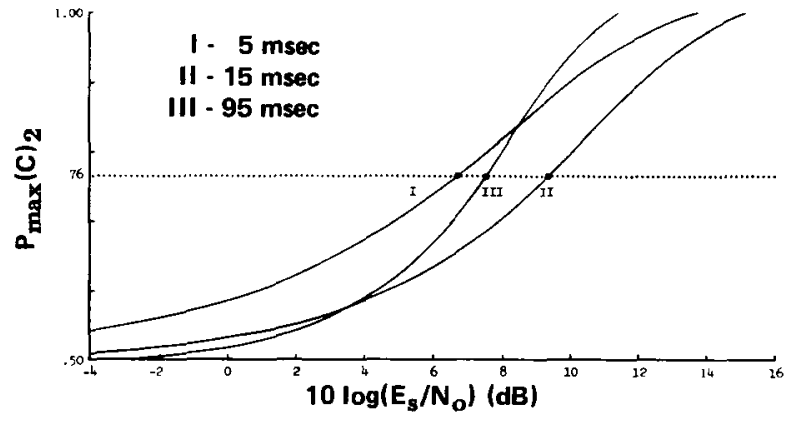

Figure 3. Complete psychometric functions whose thresholds and slopes would be those values of Figure 1 and Figure 2 for noise-fringe durations of 5,15 , and 95 msec. $P_{\max }(C)_{2}$ is the percentage of correct responses to be expected in a two-interval forcedchoice psychophysical task. (The slopes are those that would be characteristic for the linear psychometric functions obtained when the ordinate is transformed to $10 \log \mathrm{d}^{\prime}$.)

Figure 3 shows sample psychometric functions with the threshold defined at $76 \%$ correct for each. Note that joint increase in threshold and slope implies that the decrement in performance is relatively largest for weak signals as compared with strong signals, while an increase in slope coupled with some decrease in threshold suggests a relative enhancement of the detection of strong signals.

To make sense of our data, we suggest the following argument. Temporal uncertainty, that is, some measure of the extent of the receiver's lack of knowledge concerning when to read out a value from the integrator, should lead to a greater depression of performance for weak signals, since strong signals may "peak" the integrator sufficiently often to cue the detector about their existence and temporal location. Consider noise-fringe durations less than $t_{p}$, where small signals are already very difficult to detect because of temporal uncertainty. Assume that large signal peaking of the integrator is somehow masked so that the upper region of the psychometric function is suppressed; the function is shallower than would otherwise be expected. Note that the indeterminate contribution of internal noise would complicate determination of expected levels and rates of increase of both threshold and slope as noise-fringe duration approaches $t_{p}$. The threshold and slope should both be increasing more slowly than might be expected from their magnitudes alone, if the rates of increase were due solely to increasing temporal uncertainty, because a region of high uncertainty will already have been reached. This may not be apparent from the moderately low slopes. Now, assume that the "onset" and "offset" transients of the noise masker serve as excellent time markers. Noise transients close in time to the signal interval, however, may also mask the large signal peaks. Neural peak responses to transients, particularly “on” responses (Elliott, 1965; 
Green, 1969; Zwicker, 1965) may be responsible. Such masking may occur while those same transients are aids to detection as precision time markers. Consequently, temporal uncertainty regarding signal location would continue to grow as the noise transients become sufficiently distant from the signal, but while they are still the most precise time marking events available to the detector. However, this temporal uncertainty should reach a maximum as the transients move further from the signal interval, particularly because we assume the detector to have some other time masking mechanism in the context of the experiment, which would then be more dependable than the noise-burst transients. The data show that as noise-fringe duration grows beyond $t_{p}$, the signal threshold actually decreases. Our inference is that temporal uncertainty is then maximized, and also that the noise burst endpoints are so far from the signal interval that they are rapidly becoming less effective as maskers of the strong signal peaks. Consequently, strong signals will be detected with increasing probability, while the probability of detecting weak signals remains relatively constant. Thus the signal detection threshold will be decreasing as the slope of the psychometric function increases. All this provides an excellent qualitative description of the pattern of changes in the psychometric functions derived from the data.

Penner chose the peak value of $y(t)$ as the decision variable. However, neither stimulus nor biological variability was represented, and the expectation of $y(t)$, rather than $y(t)$, was used as the argument. It can be assumed that the interval with the largest momentary value of $y(t)$ is chosen as that containing the signal. Penner's argument suggests that the interval judged to contain the signal is the one with the largest expected peak, presumed to occur near the center of the interval for the case of long maskers. Penner's formal argument is a first step in a model of detection based on the peak of $y(t)$, but statistical variation must be considered in order to avoid predictions of errorless performance. Furthermore, to extend Penner's analysis requires consideration of the variability of the temporal location of the maximum of $y(t)$ as well as of the magnitude of $y(t)$, since the location of the maximum of $y(t)$ may be as valuable a cue as its size.

Our analysis also led to the conclusion that the peaks of $y(t)$ which may occur within the observation interval when the signal is present may be used by the listener, and that this mechanism is probably impor- tant for long masker durations. In this regard, our argument is similar to Penner's. However, our interpretation depended on analysis of psychometric function slopes and statistical variance of the detection process.

We point out in closing that if the formal mathematical arguments lead to one which considers both the selection of prominent peaks of the integrator and statistical variance, it is still conceivable that the results of a power integration model might be at least qualitatively compatible with our slope and threshold data. That is concluded by noting that the variance of the energy detection process is an increasing function of noise duration. If the integrator is often read out at an earlier point in time during the duration of the masker when the signal has been added, then the variance may be greater when signal is absent than when it is present. Consequently, it is conceivable that the slope of the psychometric function could grow beyond unity as masker duration increases. This can occur as the threshold is declining, as previously argued. In other words, when the signal is large enough to peak the integrator sufficiently so that the peak is read out near the temporal center of the masker, the signal-plus-noise distribution governing such trials might be significantly narrower than otherwise.

\section{REFERENCES}

Egan, J. P., Lindner, W. A., \& McFadden, D. Masking-level differences and the form of psychometric function. Perception \& Psychophysics, 1969, 4, 209-315.

ElliotT, L. L. Changes in the simultaneous masked thresholds of brief tones. Journal of the Acoustical Society of America, $1965,38,738-746$.

Green, D. M. Masking with continuous and pulsed sinusoids. Journal of the Acoustical Society of America, 1969, 46, 939-946.

Green, D. M., \& Swets, J. A. Signal detection theory and psychophysics. New York: Wiley, 1966.

Penner, M. J. Persistence and integration: Two consequences of a sliding integrator. Perception \& Psychophysics, 1975, 18, 114-120.

Penner, M. J. Erratum: Persistence and integration: Two consequences of a sliding integrator. Perception \& Psychophysics, 1976, 19, 469-470.

Tzuo, P. L. Effects of duration of fringe of noise and fringe of silence on detection of brief signals masked by noise. Unpublished doctoral dissertation, City University of New York, 1980.

Zwicker, E. Temporal effects in simultaneous masking by whitenoise bursts. Journal of the Acoustical Society of America, $1965,37,653-663$.

(Manuscript received November 18, 1980; revision accepted for publication June 22,1981 .) 